\title{
Is Initiating NOACs for Atrial Arrhythmias Safe in Adults with Congenital Heart Disease?
}

\author{
Hayang Yang ${ }^{1,2}$ (D) Berto J. Bouma ${ }^{1} \cdot$ Barbara J. M. Mulder ${ }^{1,2} \cdot$ On behalf of The Non \\ vitamin $\mathrm{K}$ antagonist Oral anticoagulants for ThromboEmbolic prevention in adult \\ congenital heart disease (NOTE) investigators
}

Published online: 7 August 2017

(C) The Author(s) 2017. This article is an open access publication

\begin{abstract}
Background In recent years, non-vitamin $\mathrm{K}$ antagonist (VKA) oral anticoagulants (NOACs) have been increasingly prescribed to adults with congenital heart disease (CHD) and atrial arrhythmias without good evidence for either safety or efficacy. To address this gap, we initiated an ongoing prospective global registry (NOTE: non-vitamin K antagonist oral anticoagulants for thromboembolic prevention in patients with congenital heart disease). Using the NOTE registry data, the present study aimed to evaluate the occurrence of any adverse events during the initiation phase (first 30 days) of NOACs in adults with CHD and atrial arrhythmias.

Methods and Results For this prospective observational study, 99 adults with CHD and atrial arrhythmias (median age 49 years [IQR 38-61], 53\% male) who initiated NOACs at or after the inclusion point were analysed. Thromboembolic events, major bleeding and other minor adverse events were assessed after the first 30 days since the initiation of NOACs. In 54 patients transitioning from VKA to NOACs, 8 minor adverse events (5 minor bleeding; 3 side-effects; 1 drop-out due to minor bleeding) occurred within 30 days after the transition. No adverse events were reported in 46 VKA-naive patients within 30 days after the initiation of NOACs.
\end{abstract}

Electronic supplementary material The online version of this article (doi:10.1007/s10557-017-6745-y) contains supplementary material, which is available to authorized users.

Barbara J. M. Mulder

b.j.mulder@amc.uva.nl

1 Department of Cardiology, Academic Medical Center, University of Amsterdam, Room B2-240, Meibergdreef 9, 1105

AZ Amsterdam, The Netherlands

2 Interuniversity Cardiology Institute of the Netherlands-Netherlands Heart Institute, Utrecht, The Netherlands
Conclusions Initiation of NOACs and transition from VKA to NOACs seem to be safe during the first month, without major adverse events and with only limited minor side effects in adults with CHD and atrial arrhythmias. This global ongoing prospective registry enables precise collection of important clinical information in real-world adults with $\mathrm{CHD}$, managed with NOACs.

Keywords Congenital heart disease $\cdot$ Atrial arrhythmia . Anticoagulation $\cdot$ Non vitamin $\mathrm{K}$ antagonist oral anticoagulant $\cdot$ Thromboembolic event $\cdot$ Bleeding

\section{Introduction}

Atrial arrhythmias are prevalent in $15 \%$ of adults with congenital heart defects (CHD) and are associated with increased risk for thromboembolism [1]. The most recently introduced oral anticoagulants, non-vitamin $\mathrm{K}$ antagonist oral anticoagulants (NOACs) have been shown to be associated with an equal or reduced risk for thromboembolism and major bleeding compared with vitamin $\mathrm{K}$ antagonist (VKA) in the general population with non-valvular atrial arrhythmias [2]. However, it is uncertain whether results from that population are applicable in CHD patients, since hearts of CHD patients are structurally different. Nevertheless, NOACs are attractive alternatives for VKA in this group of predominantly young patients, as they may be compromised in their daily life by ongoing international normalized ratio (INR) monitoring and dose adjustments. To date, few data have been published on the rates of thromboembolism and major bleeding under the use of NOACs in adults with CHD with atrial arrhythmias [3]. The utility of these data are hampered by a limited sample size and a short follow-up period. 
Accordingly, safety of NOACs needs to be assessed in adults with CHD. For this purpose, we initiated a worldwide prospective registry of efficacy and safety of non-vitamin K antagonist oral anticoagulants for thromboembolic prevention in patients with congenital heart disease; NOTE registry (https://note.reports.nl; ClinicalTrials.gov registration: NCT02928133). We present our first data on the safety of initiation of NOACs and transition from vitamin $\mathrm{K}$ antagonists to NOACs during the first 30 days in adult CHD patients with atrial arrhythmias.

\section{Methods}

In the NOTE registry, adults with CHD using NOACs for thromboembolic prevention have been enrolled from April 2014 onward. The NOTE study protocol conforms to the ethical guidelines of the 1975 Declaration of Helsinki and was approved by research committees of all participating medical centres. Informed consent was obtained from all individual participants included in the study.

This is a prospective cohort study, using data obtained from the NOTE registry. We analysed all patients using NOACs for atrial arrhythmias. We excluded the patients who initiated NOACs prior to the time of enrolment or used NOACs for other indications than atrial arrhythmias. The patients were included as they presented at the participating institutions or were identified by using a national registry. The choice of NOAC type was at the discretion of the patient's cardiologist without a specific protocol. At inclusion, demographics and pre-defined clinical data were collected. The time period of evaluation of the safety of NOACs initiation was set as within 30 days after the initiation of NOACs. Follow-up took place at $180 \pm 28$ days, 1 year \pm 28 days, and 2 years \pm 28 days, coinciding with their routine outpatient clinical visit or telephone contact. At such follow-up point, evaluation of pre-defined end points was conducted by an investigator or a site coordinator, who was trained on the protocol of the NOTE registry. The major adverse events were thromboembolism (ischemic cerebrovascular accident iCVA, transient ischemic attack TIA, systemic or pulmonary embolism or intracardiac thrombosis) and major bleeding (significant bleeding necessitating hospitalization/interventions/ $\geq 2$ units of packed cells, and/or with a haemoglobin drop $>1.24 \mathrm{mmol} / \mathrm{L}$ and/or bleeding that was fatal or occurred in the following critical sites: intra-cranial, intra-spinal, intra-ocular, pericardial, intra-articular, intramuscular with compartment syndrome) according to the International Society on Thrombosis and Haemostasis criteria [4]. As secondary endpoints, we registered other adverse events (minor bleeding event, mortality, hospitalization, interventions).

The VKA-experienced group consisted of the patients who used VKA at the time of transitioning to NOAC. The rest of the group was defined as the VKA-naive group. Any differences between the VKA-experienced group and VKA-naive group, and between the group with minor bleeding and the group without minor bleeding were analysed using unpaired ttests and reported as median with IQR or frequencies in $\%$ as appropriate. Analyses were performed with SPSS version 21.0 (IBM Corp., Armonk, NY, USA). A $p$-value below 0.05 was considered statistically significant.

\section{Results}

Through collaboration with the International Society for Adult Congenital Heart Disease (ISACHD), there are currently 31 participating medical institutions worldwide and enrolment has started in 26 of these institutions for this ongoing multicenter prospective registry. So far, 245 adults using NOACs were recruited in the NOTE registry.

For the present study, 146 patients were excluded from the analysis according to the exclusion criteria. A total of 99 adults (Table 1; median age 49 years [IQR 38-61], 53\% male) represent the study cohort for this analysis. The cohort consists of a wide variety of CHD types (Fig. 1; complex 29\%, moderate $56 \%$, simple $15 \%$ ). At the time of transition to NOACs (=baseline), $41 \%$ of the patients were not on any anti-thrombotic therapy, $4 \%$ were on aspirin and $55 \%$ used VKA (2 patients concomitant aspirin; median treatment period 7.7 years [IQR 2.5-10.6]). All patients started NOACs (apixaban 62\%; rivaroxaban 29\%; dabigatran 9\%) for atrial arrhythmias. The most common types of atrial arrhythmias were atrial fibrillation $(60 \%)$ and atrial flutter or intra atrial re-entry tachycardia (44\%). Regarding the $\mathrm{CHA}_{2} \mathrm{DS}_{2}$-VASc and HASBLED score, $57 \%$ and $88 \%$ of the patients had a score of 0 or 1, respectively. Overall, $11 \%$ had Fontan circulation (atriopulmonary connection $n=6$; lateral tunnel or intracardiac conduit $n=4$; extracardiac conduit $n=1$ ), $7 \%$ pulmonary hypertension and $33 \%$ had a history of heart failure defined by clinical signs or low systolic function by imaging (Table 1). Twelve percent of the patients previously experienced an iCVA or TIA, 3\% deep venous thrombosis, $3 \%$ intracardiac thrombosis, $2 \%$ pulmonary embolism, $1 \%$ myocardial infarction, and $9 \%$ had prior major bleeding. Compared with the VKA-naive group, the VKAexperienced group had higher HASBLED score (median 0 [IQR $0-1$ ] vs. 1 [IQR $0-1$ ], $p=0.002$ ) and higher prevalence of prior major bleeding $(2.2 \%$ vs. $14.8 \%, p=0.030)$.

Regarding the safety during the first 30-day follow-up after initiation of NOACs, none of the patients experienced a thromboembolic event or a major bleeding complication, either in the VKA-naive group or in the VKA-experienced group. In the VKA-experienced group $(n=54), 9 \%$ of the patients reported minor bleeding, most often epistaxis $(n=3)$, hematoma $(n=1)$ and gingival bleeding $(n=1)$, within 
Table 1 Baseline characteristics

\begin{tabular}{lllll}
\hline & All $(\boldsymbol{n}=\mathbf{9 9})$ & VKA $(\boldsymbol{n}=\mathbf{5 4})$ & VKA-naive $(\boldsymbol{n}=\mathbf{4 5})$ & $\boldsymbol{p}$-value \\
\hline Age at inclusion, y & $48.8(38-61)$ & $47.3(38-61)$ & $52.0(37-61)$ & 0.784 \\
Male, n(\%) & $52(53)$ & $24(44)$ & $28(62)$ & 0.078 \\
Severity of congenital heart defect, n (\%) & & & \\
Simple & $15(15)$ & $8(15)$ & $7(16)$ & 0.918 \\
Moderate & $55(56)$ & $28(52)$ & $27(60)$ & 0.417 \\
Complex & $29(29)$ & $18(33)$ & $11(24)$ & 0.333 \\
Fontan circulation & $11(11)$ & $9(17)$ & $2(4)$ & 0.054 \\
Pulmonary hypertension & $7(7)$ & $3(6)$ & $4(9)$ & 0.519 \\
Median CHA 2 DS 2 -VASc & $1(0-2)$ & $1(0-2)$ & $1(0-2.5)$ & 0.632 \\
Median HASBLED & $0(0-1)$ & $1(0-1)$ & $0(0-1)$ & 0.002 \\
Cardiovascular history, n(\%) & & & & \\
Stroke or TIA & $12(12)$ & $7(13)$ & $5(11)$ & 0.779 \\
Pulmonary embolism & $2(2)$ & $1(2)$ & $1(2)$ & 0.941 \\
Deep venous thrombosis & $3(3)$ & $1(2)$ & $2(4)$ & 0.454 \\
Intracardiac thrombosis & $3(3)$ & $3(6)$ & 0 & 0.095 \\
Myocardial infarction & $1(1)$ & $1(1)$ & 0 & 0.359 \\
Major bleeding & $9(9)$ & $8(15)$ & $1(2)$ & 0.030 \\
Heart failure* & $33(33)$ & $20(37)$ & $13(29)$ & 0.392 \\
Hypertension & $24(24)$ & $11(20)$ & $4(9)$ & 0.319 \\
Diabetes mellitus & $8(8)$ & $4(7)$ & 0.788 \\
\hline
\end{tabular}

Values are presented as median (IQR) or counts $(\%)$. *Heart failure is defined as the presence of signs and symptoms of either right (elevated central venous pressure, hepatomegaly, dependent oedema) or left ventricular failure (exertional dyspnoea, cough, fatigue, orthopnoea, paroxysmal nocturnal dyspnoea, cardiac enlargement, crackles, gallop rhythm, pulmonary venous congestion) or both, confirmed by non-invasive or invasive measurements demonstrating objective evidence of cardiac dysfunction

Abbreviations: VKA, vitamin K antagonist-experienced group; VKA-naive, vitamin K antagonist naive group; CHA2DS2-VASc, stroke risk factor scoring system in which 1 point is given for heart failure, hypertension, age 64-74 years, diabetes mellitus, history of vascular disease, female sex and 2 points are given for age $\geq 75$ years, history of stroke/TIA/thromboembolism; HASBLED, bleeding risk factor scoring system in which 1 point is given for uncontrolled hypertension, abnormal renal or liver function, history of stroke or bleeding, labile international normalized ratio, age $>65$ years, use of nonsteroidal anti-inflammatory drug or antiplatelet agents or alcohol; NOAC, new oral anticoagulant; TIA, transient ischemic attack

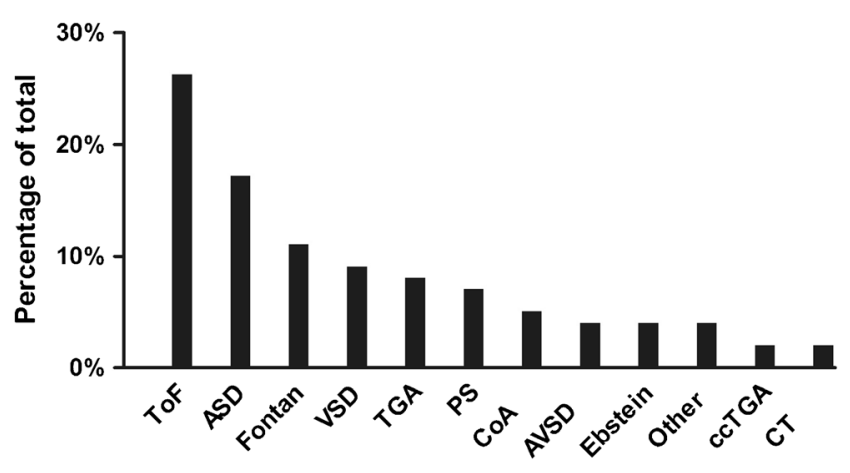

Fig. 1 Distribution of congenital heart defects. Abbreviations: ToFtetralogy of Fallot; ASD-atrial septal defect; Fontan-Fontan circulation; VSD-ventricular septal defect; TGA-transposition of the great arteries; PS-pulmonary valve stenosis; CoA- aortic coarctation; AVSDatrioventricular septal defect; Ebstein-Ebstein's anomaly; Other- doubleoutlet right ventricle, pulmonary atresia, bicuspid aortic valve, Marfan syndrome; ccTGA-congenitally corrected transposition of the great arteries; CT-cor triatriatum the first 30 days after the transition. Due to frequent epistaxis, a 42-year old female with coarctation of the aorta, ceased NOAC therapy within 30 days and switched back to VKA. Other side effects such as dizziness, headaches, nausea and weakness were also reported $(n=3)$ in this group. All patients who reported minor adverse events $(n=8)$ were in the VKA-experienced group and were relatively young $(<65 \mathrm{yr})$ and had moderate or severe CHD; none had a Fontan circulation. No adverse events were reported by patients in the VKA-naive group $(n=45)$. The prevalence of higher HASBLED score $(\geq 2)$ was similar between the group with minor bleeding and the group without minor bleeding $(12.8 \%$ vs. $0 \%, p=0.394)$.

\section{Discussion}

Initiation of NOACs and transition from VKA to NOACs appeared to be safe for at least the short-term, without major 
adverse events and with limited minor side effects in 99 adults with CHD and atrial arrhythmias.

Current guidelines state that NOACs are only considered in patients with a simple form of CHD due to lack of data in patients with moderate or complex forms of CHD [5]. Our findings show reassuring short term safety results in a cohort largely consisting of moderate or complex forms of CHD $(85 \%)$, including patients with Fontan circulation (11\%) who are prone to thromboembolism and bleeding. This result may be due to the low median $\mathrm{CHA}_{2} \mathrm{DS}_{2}$-VASc score, HASBLED score and young age in this cohort. Furthermore, recent studies show mixed results on the validity of $\mathrm{CHA}_{2} \mathrm{DS}_{2}$-VASc and HASBLED scores in adults with CHD and atrial arrhythmias $[6,7]$. However, due to the presence of their structural heart defects, adults with CHD are considered to have other risk factors for stroke than just the $\mathrm{CHA}_{2} \mathrm{DS}_{2}$-VASc score.

Notably, all patients with minor adverse events were VKAexperienced and had moderate to severe type of CHD. This may be related to the higher HASBLED score and higher prevalence of prior major bleeding in the VKA-experienced group compared with the VKA-naive group. However, a higher HASBLED score $(\geq 2)$ at baseline was not related to higher minor bleeding risk during the follow up.

This study is limited by the modest sample size, number of events over a short-term follow-up and the observational design. Therefore, the data should be interpreted with caution pending validation in other cohorts with larger sample sizes. Furthermore, due to the low number of events in this study, we were unable to perform regression analysis to determine whether CHD complexity is a predictor of adverse events. We expect to address many of these limitations in the future with this ongoing global registry and welcome new participants to join us (https://note.reports.nl).

\section{Conclusions}

Initiation of NOACs and transition from VKA to NOACs seem to be safe during the first month, without major adverse events and with limited minor side effects in adults with CHD and atrial arrhythmias. This global ongoing prospective registry enables precise collection of important clinical information in real-world adults with CHD, managed with NOACs.

Acknowledgements We thank T. Kronvall, A. Proietti and R. Bolanos for their assistance in patient inclusion, data collection and study administration.

The NOTE investigators:

J.F. Heidendael, $\mathrm{MD}^{1,2}$, G. Veen, $\mathrm{MD}, \mathrm{PhD}^{3}$, T.C. Konings, $\mathrm{MD}^{3}$, G.T.J. Sieswerda, $\mathrm{MD}, \mathrm{PhD}^{4}$, F.J. Meijboom, $\mathrm{MD}, \mathrm{PhD}^{4}$, M.C. Post, $\mathrm{MD}, \mathrm{PhD}^{5}$, A. van Dijk, MD, $\mathrm{PhD}^{6}$, W. Budts, MD, $\mathrm{PhD}^{7}, \mathrm{M}$. Morissens, $\mathrm{MD}^{8}$, M. Ladouceur, $\mathrm{MD}^{9}$, D. Tobler, $\mathrm{MD}^{10}, \mathrm{M}$. Schwerzmann, MD ${ }^{11}$, T. Rutz, $\mathrm{MD}^{12}$, J. Bouchardy, $\mathrm{MD}^{13}, \mathrm{M}$. Greutmann, $\mathrm{MD}^{14}$, G. Scognamiglio, $\mathrm{MD}, \mathrm{PhD}^{15}$, K. Skoglund, MD, $\mathrm{PhD}^{16}$, C. Christersson, $\mathrm{MD}, \mathrm{PhD}^{17}$, L. Gumbiene, $\mathrm{MD}, \mathrm{PhD}^{18}, \mathrm{M}$.
Laukyte, $\mathrm{MD}^{18}$, P. Khairy, MD, $\mathrm{PhD}^{19}$, J. Aboulhosn, $\mathrm{MD}^{20}$, G. Veldtman, $\mathrm{MD}^{21}$, G. Webb, $\mathrm{MD}^{21}$, C.S. Broberg, $\mathrm{MD}^{22}$, A.R. Opotowsky, MD, $\mathrm{MPH}^{23}$, K. Shafer, $\mathrm{MD}^{23}$, S.F. Tsai, $\mathrm{MD}^{24}$, T. Moe, $\mathrm{MD}^{25}, \mathrm{~K}$. Niwa, $\mathrm{MD}, \mathrm{PhD}^{26}$, A. Mizuno, $\mathrm{MD}^{26}$.

1. Department of Cardiology, Academic Medical Centre, Amsterdam, the Netherlands.

2. Interuniversity Cardiology Institute of the Netherlands Netherlands Heart Institute, Utrecht, the Netherlands.

3. Department of Cardiology, VU University Medical Centre, Amsterdam, the Netherlands.

4. Department of Cardiology, University Medical Centre Utrecht, Cardiology, Utrecht, the Netherlands.

5. Department of Cardiology, St. Antonius Hospital, Nieuwegein, the Netherlands.

6. Department of Cardiology, Radboud University Nijmegen Medical Centre, Nijmegen, the Netherlands.

7. Department of Cardiology, University Hospitals Leuven, Leuven, Belgium.

8. Department of Cardiology, Brugmann University Hospital, Brussels, Belgium.

9. Department of Cardiology, Hôpital Européen Georges Pompidou, Université Paris Descartes, Paris, France.

10. Department of Cardiology, University Hospital Basel, Basel, Switzerland.

11. Department of Cardiology, Bern University Hospital, Bern, Switzerland.

12. Department of Cardiology, University Hospital Centre Vaudois (CHUV), Lausanne, Switzerland.

13. Department of Cardiology, University Hospital Geneva, Genève, Switzerland.

14. Department of Cardiology, University Hospital Zurich, Zurich, Switzerland.

15. Department of Cardiology, Vincenzo Monaldi Hospital, Naples, Italy.

16. Department of Cardiology, Sahlgrenska University Hospital, Gothenburg, Sweden.

17. Department of Cardiology, Uppsala University Hospital, Uppsala, Sweden.

18. Department of Cardiology, Vilnius University Hospital, Vilnius, Lithuania.

19. Department of Cardiology, Montreal Heart Institute, Montreal, Canada.

20. Department of Cardiology, Ronald Reagan UCLA Medical Centre, Los Angeles, United States of America.

21. Department of Cardiology, Cincinnati Children's Hospital Medical Centre, Cincinnati, United States of America.

22. Department of Cardiology, Oregon Health \& Science University Hospital, Portland, United States of America.

23. Department of Cardiology, Boston Children's hospital, Boston, United States of America.

24. Department of Cardiology, University of Nebraska Medical Centre, Nebraska, United States of America.

25. Department of Cardiology, Phoenix Children's Heart Centre, Phoenix, United States of America.

26. Department of Cardiology, St. Luke's International Hospital, Tokyo, Japan.

\section{Compliance with Ethical Standards}

Funding The Work Described in this Study was Carried Out in the Context of the Parelsnoer Institute (PSI). PSI is Part of and Funded by the Dutch Federation of University Medical Centres. This Work is Supported by Restricted Research Grants from Bristol-Myers Squibb, Ingelheim-Boehringer, Bayer, and Daiichi Sankyo. These Companies 
had No Role in Data Collection, Analysis, or Interpretation, or in the Decision to Submit this Article for Publication.

Conflict of Interest $\quad$ Dr. C. Christersson has received speaker fees from Bristol Myers Squibb, CSL Behring and Novartis, and advisory board fees from Boehringer Ingelheim. Dr. B.J. Bouma has received restricted research grant from Bristol-Myers Squibb. Dr. B.J.M. Mulder has received restricted research grants from Ingelheim-Boehringer, Bayer, and Daiichi Sankyo. Authors, J.F. Heidendael, G. Veen, T.C. Konings, G.T.J. Sieswerda, F.J. Meijboom, M.C. Post, A. van Dijk, W. Budts, M. Morissens, M. Ladouceur, Tobler, M. Schwerzmann, T. Rutz, J. Bouchardy, M. Greutmann, G. Scognamiglio, K. Skoglund, C. Christersson, L. Gumbiene, M. Laukyte, P. Khairy, J. Aboulhosn, G. Veldtman, G. Webb, C.S. Broberg, A.R. Opotowsky, MD, K. Shafer, S.F. Tsai, T. Moe, K. Niwa, A. Mizuno declare that they have no conflict of interest.

Ethical Approval All procedures performed in studies involving human participants were in accordance with the ethical standards of the institutional and/or national research committee and with the 1964 Helsinki declaration and its later amendments or comparable ethical standards.

Informed Consent Informed consent was obtained from all individual participants included in the study.

Open Access This article is distributed under the terms of the Creative Commons Attribution 4.0 International License (http:// creativecommons.org/licenses/by/4.0/), which permits unrestricted use, distribution, and reproduction in any medium, provided you give appropriate credit to the original author(s) and the source, provide a link to the Creative Commons license, and indicate if changes were made.

\section{References}

1. Bouchardy J, Therrien J, Pilote L, et al. Atrial arrhythmias in adults with congenital heart disease. Circulation. 2009;120:1679-86.

2. Ruff CT, Giugliano RP, Braunwald E, et al. Comparison of the efficacy and safety of new oral anticoagulants with warfarin in patients with atrial fibrillation: a meta-analysis of randomised trials. Lancet. 2014:383:955-62.

3. Pujol C, Niesert A-C, Engelhardt A, et al. Usefulness of direct oral anticoagulants in adult congenital heart disease. Am J Cardiol. 2016;117:450-5.

4. Rodeghiero F, Tosetto A, Abshire T, et al. ISTH/SSC bleeding assessment tool: a standardized questionnaire and a proposal for a new bleeding score for inherited bleeding disorders. J Thromb Haemost. 2010;8:2063-5.

5. Khairy P, Van Hare GF, Balaji S, et al. PACES/HRS expert consensus statement on the recognition and Management of Arrhythmias in adult congenital heart disease: developed in partnership between the pediatric and congenital electrophysiology society (PACES) and the Heart Rhythm Society (HRS). En Heart Rhythm. 2014;11:e102-65.

6. Heidendael JF, Bokma JP, de Groot JR, et al. Weighing the risks: thrombotic and bleeding events in adults with atrial arrhythmias and congenital heart disease. Int J Cardiol. 2015;186:315-20.

7. Khairy P, Aboulhosn J, Broberg CS, et al. Thromboprophylaxis for atrial arrhythmias in congenital heart disease: a multicenter study. Int J Cardiol. 2016;223:729-35. 